\title{
Perfil y concepción de la enseñanza del entrenador de fútbol base de la región de Beja (Portugal) \\ Profile and conception of the teaching of the basic football coach in the region of Beja (Portugal) \\ *Paulo Paixao, **Fco. Javier Giménez Fuentes-Guerra, **Benjamin Navarro Domínguez, **Jose Antonio Cerrada Nogales, **José Robles Rodríguez, **Manuel Tomás Abad Robles \\ *Instituto Politécnico de Beja (Portugal), **Universidad de Huelva (España)
}

Resumen: La figura del entrenador es clave en el entrenamiento deportivo con jóvenes. Este estudio tuvo como objetivos establecer el perfil del entrenador de fútbol en las etapas de formación de los jóvenes jugadores de la región de Beja (Portugal), identificar la concepción sobre la enseñanza del fútbol que tienen, y conocer la forma de planificar y programar de los mismos. Con este fin se encuestaron a 124 entrenadores de fútbol base de la mencionada región portuguesa. Los datos mostraron que el perfil del entrenador de fútbol base de Beja se correspondió al siguiente: hombre de entre 31 y 40 años, con el duodécimo año de escolaridad completo, trabajando por cuenta ajena, sin curso de entrenador o con apenas el Grado I o nivel básico de la titulación de entrenador de fútbol. También destacó el hecho de que la mayoría de los entrenadores consideraran el fútbol un medio para educar a los jugadores, siendo los entrenadores más jóvenes los que más creían en el fútbol como medio transmisor de valores. Cabe destacar que los entrenadores manifestaron tener bastante claro los contenidos a trabajar en estas edades y que solían utilizar tanto métodos de enseñanza directivos como basados en el descubrimiento. Los entrenadores afirmaron que solían dar más prioridad a los aspectos técnico-tácticos con respecto al trabajo físico. Por último, gran parte de los entrenadores mostraron intención de entrenar en un futuro en categorías superiores.

Palabras clave: Entrenador de fútbol, enseñanza del deporte, planificación y programación, formación jugadores.

\begin{abstract}
The figure of the coach is key in sports training with young people. The aims of this study were to establish the profile of the football coach in the training stages of young players in the region of Beja (Portugal), to identify their conception of football teaching, and to know how to plan and programme them. To this end, 124 grassroots football coaches in the aforementioned Portuguese region were surveyed. The data showed that the profile of the base football coach in Beja corresponded to the following: a man between 31 and 40 years old, with the twelfth year of schooling complete, working as an employee, without a course of coaching or with only Grade I or basic level of the qualification of football coach. It was also highlighted the fact that most coaches considered football as a means of educating players, with younger coaches believing most in football as a means of transmitting values. It should be noted that the coaches said they were quite clear about the content to be worked on in these ages and that they used both managerial and discovery-based teaching methods. Coaches said they tended to give more priority to technical and tactical aspects over physical work. Finally, a large number of coaches showed their intention to train at higher levels in the future.
\end{abstract}

Key words: Football coach, sports teaching, planning and programing.

\section{Introducción}

El entrenador es clave en el entrenamiento deportivo con jóvenes, constituyéndose como una de las fuerzas más influyentes en el correcto desarrollo de los niños y niñas (Evans et al., 2015; Maestre et al., 2018; Lledó, Martínez \& Huertas, 2014; Santos et al., 2016). Entre los técnicos deportivos, suelen abundar aquéllos que pertenecen al género masculino, con estudios básicos y con poca experiencia (Gutiérrez, 2007; Lledó et

Fecha recepción: 24-01-21. Fecha de aceptación: 25-03-21

Fco. Javier Giménez Fuentes-Guerra

jfuentes@uhu.es al., 2014; Nuviala et al., 2007). Además, cabe destacar que, entre las causas más destacadas del abandono de la práctica deportiva por parte de los jóvenes, se encuentra la conducta inadecuada del entrenador (Macarro, Romero \& Torres, 2010), por lo que una correcta conducta de éste, junto con una adecuada concepción de la enseñanza deportiva, pueden contribuir a una disminución del abandono de dicha práctica (Nuviala \& Nuviala, 2005; Sousa et al., 2006).

De esta manera, en la adquisición de una concepción adecuada de la enseñanza y del entrenamiento del fútbol con jóvenes, resulta fundamental la formación de los entrenadores. Al respecto, Azpillaga et al. (2012) plantean que los entrenadores deberían sentirse atraí- 
dos por la mejora continua de su formación y por la actualización de sus conocimientos. Uno de los aspectos claves en la formación deportiva de jóvenes es la concepción que debe tener el entrenador de la competición, la cual ha de contemplarse como un escenario socioeducativo que garantice que el referente educativo sobresalga respecto al enfoque puramente competitivo (Merino, Sabirón, \& Arraiz, 2015). En contraposición, a la hora de valorar la eficacia de los entrenamientos y el nivel de aprendizaje adquirido por los jugadores, se suele tener en cuenta, fundamentalmente, los resultados obtenidos en las competiciones, en detrimento de la consideración de la competición como un medio de aprendizaje. Sin embargo, es el análisis del proceso de planificación e intervención en el entrenamiento el encargado de facilitar información referida a la adecuación del mismo y a su posible reorientación $(\mathrm{Ca}-$ ñadas et al., 2010). En este sentido, el estudio del desempeño profesional de los entrenadores de fútbol base es fundamental para llevar a cabo análisis adecuados de los procesos de competición y de los resultados deportivos obtenidos (Ballester, Fernández y Parra-Camacho, 2021).

Por otro lado, la planificación del entrenamiento deportivo es un proceso complejo y creativo, que requiere tiempo y dedicación. En las últimas dos décadas, la planificación ha suscitado un gran interés en la literatura de las ciencias del deporte, aunque actualmente no se ha podido definir y estudiar con claridad y exactitud, lo cual puede llevar a cierto desacuerdo sobre los componentes específicos que deben formar parte de la misma (Kataoka et al., en prensa). No obstante, Bettega et al. (2018) consideran importante que el entrenador sea consciente y comprenda el proceso de planificación, el entrenamiento y el juego. En este sentido, se recomienda que los entrenadores lleven a cabo la planificación empezando con el diseño de un plan anual sólido que sirva de hoja de ruta para implementar los entrenamientos (Suchomel et al., 2018). Al respecto, Abad (2009) señala la importancia de planificar el trabajo a desarrollar señalando las siguientes ventajas: la seguridad, el orden, la variedad, eficiencia, mejora y coordinación del trabajo. A través de la planificación de los entrenamientos de una temporada o periodo, los objetivos van estableciéndose fruto de un proceso reflexivo e intencionado. Además, la planificación del entrenador puede ser analizada desde un punto de vista tradicional o desde una concepción alternativa o comprensiva de la enseñanza-aprendizaje del fútbol. La elección de una u otra perspectiva va a influir notablemente en el proceso formativo de los jugadores (Urbano-Arévalo et al., 2020).

En este sentido, la planificación durante la etapa de formación deportiva, debe ser diferente de la planificación de equipos de mayor nivel y categoría, ya que ésta debe basarse en la globalidad y la polivalencia (Giménez, 2003). Por su parte, Ibáñez (2002) considera los contenidos del entrenamiento como una de las variables pedagógicas que definen el entrenamiento deportivo. A su vez, los contenidos hacen referencia a la materia que se va a trabajar en el entrenamiento deportivo en cada temporada, sesión y tarea, suponiendo un escalón más en la programación del trabajo a desarrollar. Autores como Viciana (2002) dividen el proceso de programación en tres fases: fases de diagnóstico inicial, fase de ejecución protagonizada por los objetivos y fase de evaluación del proceso y del resultado, siendo conveniente que este proceso de programación llevado a cabo por el docente o entrenador deportivo quede enmarcado dentro de una unidad de enseñanza-aprendizaje (Siedentop, Hastie, \& Van Der Mars, 2004).

El estudio del entrenador puede aportar datos interesantes que mejoren su intervención y consecuentemente el proceso de aprendizaje de los jugadores (Giménez, 2003), lo cual lleva a que este tema tenga gran vigencia en la actualidad, haciendo necesario saber más acerca de quienes tienen tanta influencia sobre los jugadores de fútbol jóvenes que están en desarrollo (Jones, 1992). De esta manera, los estudios sobre la importancia del papel del entrenador han ido incrementándose (Santos et al., 2016) cobrando especial interés la concepción de enseñanza y los procesos de planificación y programación que llevan a cabo los entrenadores en estas edades. En este sentido, es necesaria la realización de más estudios al respecto. Por este motivo, los objetivos de esta investigación fueron: establecer el perfil del entrenador de fútbol en las etapas de formación de los jóvenes jugadores (6-18 años) de la región de Beja (Portugal), identificar la concepción sobre la enseñanza del fútbol que tienen, y conocer la forma de planificar y programar de los mismos.

\section{Material y método}

\section{Participantes}

En el estudio participaron un total de 124 entrenadores de jóvenes futbolistas distribuidos por las diferentes provincias de la región de Beja. Esta muestra corresponde al 91\% de los entrenadores de formación existentes en toda la región de estudio. El 98.4\% eran hombres $(n=122)$, y el $1.6 \%$ mujeres $(n=2)$. 


\section{Instrumento}

El instrumento utilizado en esta investigación fue el cuestionario para estudiar la formación de entrenadores de fútbol base validado por Paixão, Abad \& Giménez (2019). Estos autores validaron esta herramienta, en primer lugar, a través de un grupo de 12 jueces-expertos, quienes realizaron tanto un análisis cuantitativo como cualitativo de la misma. Posteriormente, lo aplicaron a una muestra piloto de 20 entrenadores con el mismo perfil de los encuestados finalmente. Además, realizaron un análisis de fiabilidad test-retest y calcularon el coeficiente de correlación intraclase. El cuestionario estaba constituido por 121 ítems agrupados en 8 dimensiones: características sociodemográficas, experiencia deportiva, aspectos personales, formación como entrenador, formación continua, concepción de enseñanza, planificación y programación y necesidades de formación. Sin embargo, por motivos de extensión, este trabajo se ha centrado en las dimensiones: características sociodemográficas, concepción de enseñanza, y planificación y programación. Se utilizó una escala Likert graduada de 1 a 10, donde 1 significaba nada de acuerdo y 10 totalmente de acuerdo. Además, también se incluyeron preguntas de elección múltiple. Respecto a la consistencia interna, los Alfa de Cronbach obtenidos fueron: dimensión concepción de enseñanza (á $=.780)$ y dimensión planificación y programación (á $=.735)$.

\section{Procedimiento}

El proceso seguido para la realización de esta investigación comenzó al contactar con la Asociación de Fútbol de Beja (AFB) para conseguir información acerca de los entrenadores de fútbol base de la región durante la temporada 2017/18. De esta forma, se obtuvieron los teléfonos, direcciones y correos electrónicos de los mismos Posteriormente, se contactó para determinar la forma de pasar el cuestionario, el lugar y el momento de proceder a su recogida, en caso de llevarse a cabo de forma presencial. . Antes de iniciar la toma de datos se informó a los participantes sobre la finalidad del estudio y se garantizó que la información recogida seguiría siendo confidencial, cumpliendo con las normas éticas y la Declaración de Helsinki, contando con el consentimiento informado de los participantes. A los entrenadores que optaron por su realización vía telemática se les envió el enlace del cuestionario a la dirección de correo electrónico para que llevaran a cabo su cumplimentación a través de internet y la información fuera recogida empleando esta misma vía.

\section{Análisis estadístico}

Se llevó a cabo un análisis descriptivo básico de tendencia central y dispersión de cada uno de los ítems. Se utilizó la prueba Kolmogorov-Smirnov para la prueba de normalidad, obteniéndose valores $p<.05$, por lo que se procedió al análisis de estadísticos no paramétricos. Para determinar si existían diferencias significativas, se usó la prueba de contraste Kruskal-Wallis, en aquellos casos en los que las comparaciones intergrupales fueron significativas $(p<.05)$, se realizó la prueba post-hoc mediante el test de Dunn para cada par de grupos, aplicando el ajuste de Bonferroni para corrección del nivel de significación. Se calculó el tamaño del efecto (Tomczak \& Tomcak, 2014), y para la valoración de los mismos se estableció el criterio descrito por Cohen (1988): $d=$ .10 (bajo), $d=.30$ (medio), $d=.50$ (grande), $d=.70$ (muy grande). El programa estadístico utilizado fue el SPSS en su versión 23.0.

\section{Resultados}

\section{Perfil de los entrenadores}

El 98.4\% eran hombres $(n=122)$, y el 1.6\% mujeres $(n=2)$. En cuanto a la edad de los participantes, el $31.5 \%$ tenía menos de 30 años $(n=39)$, el $35.5 \%$ estaba entre 31 y 40 años $(n=44)$, el 24.2\% tenía entre 41 y 50 años $(n=30)$, y el $8.9 \%$ tenía más de 50 años $(n=11)$.

Respecto a la formación académica se observó que, el 20.2\% poseía estudios primarios $(n=25)$, el $40.3 \%$ estudios secundarios, Formación Profesional o Bachillerato $(n=50)$, el 26.6\% tenía titulación universitaria en Educación Física $(n=33)$, y el $12.9 \%$ otras titulaciones universitarias $(n=16)$. Por otra parte, el 25\% de los entrenadores $(\mathrm{n}=31)$, no tenía el título de entrenador, el 33,1\% ( $n=41)$, estaba realizando los cursos de entrenador en el momento en el que se le pasó el cuestionario, con lo que solo el 58,1\% ( $=52)$, tenía titulación de entrenador de fútbol. En cuanto a la experiencia como entrenador, el 32.3\% tenía menos de 2 años $(n=40)$, el $26.6 \%$ entre 3 y 5 años $(n=33)$, el $17.7 \%$ entre 6 y 8 años $(n=22)$, y el 23.4\% llevaba 9 o más años como entrenador $(n=29)$.

Por otro lado, en relación con la experiencia como deportista, el 75\% habían sido jugadores federados $(n=$ 93), el 22\% lo seguían siendo $(n=22)$, y sólo el $7.3 \%$ no había tenido experiencia como futbolista federado ( $n$ $=7$ ). Además, se detectó que 37.9\% había jugado durante la etapa de formación deportiva (categorías juvenil o inferior) ( $n=47)$, el 38.7\% lo había hecho en ligas menores durante la etapa de senior (ligas provinciales o 


\begin{tabular}{|c|c|c|c|c|}
\hline Ítems Dimensión Concepción de Enseñanza & $M D T$ & Edad $\left(X^{2} ; p\right)$ & $\begin{array}{c}\text { Experiencia } \\
\text { entrenador }\left(X^{2} ; p\right)\end{array}$ & $\begin{array}{c}\text { Experiencia } \\
\text { jugador }\left(X^{2} ; p\right)\end{array}$ \\
\hline En mi opinión, el fútbol es un medio a través del cual puedo educar a mis jugadores & $5.98 \pm 1.980$ & $(12.535 ; .006)$ & $(8.987 ; .029)$ & - \\
\hline Para mí, los resultados obtenidos por mi equipo en las competiciones son muy importantes & $7.87 \pm 1.834$ & & - & - \\
\hline La competición es sólo un aspecto más en la formación deportiva de los jugadores & $8.65 \pm 1.568$ & & & \\
\hline Considero que lo primordial es que los jugadores se divierten practicando fútbol & $9.25 \pm .934$ & & & $(13.951 ; .001)$ \\
\hline A través del fútbol podemos transmitir valores educativos & $7.56 \pm 1.515$ & $(12.563 ; .006)$ & & \\
\hline Entiendo que debo utilizar con más frecuencia métodos de enseñanza directivos & $7.77 \pm 1.561$ & & & \\
\hline Entiendo que debo utilizar con más frecuencia métodos de enseñanza basados en el descubrimiento & $7.53 \pm 2.151$ & & & \\
\hline Dispongo con frecuencia de instalaciones y material suficiente para entrenar & $7.55 \pm 1.494$ & - & - & \\
\hline Creo que la competición en estas edades de iniciación está adaptada a las características psicoevolutivas de los jugadores & $8.79 \pm 1.589$ & - & - & - \\
\hline Creo que todos los jugadores deben participar en las competiciones, independientemente del resultado & $8.33 \pm 1.851$ & - & - & - \\
\hline
\end{tabular}
$M=$ Media; $D T=$ Desviación típica; $X^{2}=$ Chi-cuadrado $p=$ significatividad $(<05)$ Variable de contraste: KruskalWallis.

autonómicas) ( $n=48)$ y, finalmente, el 23.4\% había jugado en categorías de 2 división B o superiores $(n=29)$.

\section{Dimensión Concepción de Enseñanza}

Atendiendo a los datos obtenidos, se observó que los entrenadores consideraron que es primordial que los jugadores se diviertan practicando fútbol. En este sentido, los entrenadores que actualmente seguían practicando fútbol de manera federada valoraron más dicha afirmación respecto al resto de encuestados (entrenadores-jugadores federados en la actualidad vs federados anteriormente: $Z=2.983 ; p=.009$; tamaño del efecto medio: $r=0.3$ ), (jugadores-entrenadores federados actualmente vs no federados nunca: $Z=-3.412 ; p=.002$; tamaño del efecto grande: $r=.6$ ).

Por otra parte, en relación con los ítems referentes a la competición, todos fueron bien valorados por la mayoría de los encuestados. En este sentido, los entrenadores consideraban que la competición estaba adaptada a las características psicoevolutivas de los jugadores, que la competición era un medio más en la formación de los futbolistas, y que todos los jugadores debían participar independientemente del resultado. Finalmente, los entrenadores consideraron muy importantes los resultados obtenidos por su equipo en las competiciones.

En cuanto a la utilización de los métodos de enseñanza, se observó que ambas preguntas mostraron datos similares, tanto la referida a la utilización de métodos directivos como la utilización de métodos de enseñanza basados en el descubrimiento.

Por último, los ítems referidos al fútbol como medio educativo y como medio transmisor de valores, no fueron igualmente valorados por todos los encuestados. Respecto a la opinión de que el fútbol es un medio a través del cual se puede educar a los jugadores, los entrenadores de más de 50 años valoraron más dicho aspecto en comparación con los de entrenadores de edades comprendidas entre 31-40 años $(Z=-2.818 ; p=$ $.029)$, con un tamaño del efecto medio $(r=.4)$. Atendiendo a la experiencia como entrenador, también se observó que los entrenadores que más tiempo llevaban ejerciendo valoraron más el fútbol como medio para educar, los datos mostraron diferencias significativas (entrenadores con menos de dos años de experiencia vs entrenadores con más de nueve años de experiencia: $Z=-2.803 ; p=.030$; tamaño del efecto medio: $r=.3$ ).

Respecto al ítem que hace referencia al fútbol como medio para transmitir valores, llama la atención que, por el contrario, los entrenadores de menor edad son los que valoran más dicha afirmación, mostrando diferencias significativas con el resto de grupos (menos de 30 años vs 41-50 años: $Z=2.751 ; p=.036$; tamaño del efecto medio: $r=.3$ ) y (menos de 30 años vs 31-40 años: $Z=2.918 ; p=.021$; tamaño del efecto medio: $r=$ .3). No se detectaron diferencias significativas en ninguno de los ítems relacionados con la transmisión de valores, atendiendo a la formación académica de los entrenadores (Tabla 1).

\section{Dimensión Planificación y Programación}

En relación con el modo de programar, los datos mostraron que tanto la programación a largo plazo como la programación a corto plazo obtuvieron valores altos, siendo la programación semanal la que mayores valores presentó. En cuanto al modo de trabajar los medios técnico-tácticos, las situaciones con oposición, similares al juego real y de forma lúdica, se hallaron valores elevados, y priorizándose respecto a los aspectos físicos. Por otro lado, los datos mostraron que los entrenadores asignaron una puntuación alta a los ítems que hacían referencia a las relaciones que los entrenadores mantenían tanto con los propios deportistas como con las familias.

Por otra parte, al preguntar sobre si los entrenadores tenían claro los contenidos a desarrollar en estas edades, se encontraron diferencias significativas atendiendo a la formación académica y a la experiencia como entrenador. Respecto a la experiencia como entrenador, aquellos entrenadores que llevaban más años entrenando tenían más claros los contenidos a trabajar que los entrenadores con menor experiencia (menos de dos años de experiencia vs entre 3-5 años de experiencia: 
$Z=-2.729 ; p=.006$; tamaño del efecto medio: $r=.3$ ). Por otra parte, los entrenadores con estudios universitarios en Educación Física, también tenían más claros los contenidos a trabajar respecto a los entrenadores que sólo tenían los estudios primarios $(Z=-2.950 ; p=$ .003; tamaño del efecto medio: $r=.4$ ).

En cuanto a los objetivos, los entrenadores valoraron más en estas edades los objetivos educativos, respecto a los objetivos competitivos y recreativos. En relación con los objetivos educativos, los entrenadores de mayor edad presentaron mayores valores en comparación con los grupos de edades inferiores (menos de 30 años vs 41-50 años: $Z=-2.836 ; p=.005$; tamaño del efecto medio: $r=.3$ ) y (entre $31-40$ años vs más de50 años: $Z=-2.854 ; p=.005$; tamaño del efecto medio: $r=$ .3).

Por otro lado, respecto a la evaluación, los entrenadores dieron más valor al hecho de evaluar a principios de temporada, mientras que la evaluación al final de la temporada fue el ítem menos valorado. Finalmente, cabe señalar que el ítem que hacía referencia a la intención de los entrenadores de entrenar en un futuro en categorías superiores mostró valores elevados.

gués del Deporte y de la Juventud (2018), en el cual un $86 \%$ de entrenadores de diversas modalidades pertenecían al género masculino. En cuanto a la edad se refiere, se halló un mayor número de entrenadores entre los 31 y los 40 años, valores que se encuentran por debajo de la media, de 42 años de los entrenadores en Portugal, aportada por el Instituto Portugués del Deporte y de la Juventud (2018).

En lo referido a la cualificación académica, cabe destacar el hecho de que pocos entrenadores tenían una titulación universitaria en Educación Física y Deporte. Este hallazgo no puede ser considerado como un aspecto positivo, ya que, según Santos et al. (2010), los entrenadores con el grado superior en Educación Física y Deporte tienen una mayor percepción competencial, con respecto a aquellos sin formación superior en dicha área. Los resultados obtenidos concuerdan con la investigación de Abad et al. (2011). Por el contrario, no se encuentran en la línea de los hallados por Lledó et al. (2014), quienes concluyen que los responsables de las escuelas deportivas tienden a contratar entrenadores altamente cualificados. Además, gran parte de los entrenadores habían sido jugadores federados y tenían poca experiencia como entrenadores, lo cual coincide con lo encontrado por Abad et al. (2011). Por lo tanto, si se tiene en cuenta la titulación académica, el título de entrenador y la poca experiencia de la mayoría de los entrenadores, se puede considerar que la formación y cualificación de éstos no es la adecuada para llevar a cabo su labor como técnicos de fútbol de formación.

En general, los encuestados manifestaron un

\section{Discusión}

Los objetivos del presente estudio fueron: establecer el perfil del entrenador de fútbol en las etapas de formación de los jóvenes jugadores de la región de Beja (Portugal), identificar la concepción sobre la enseñanza del fútbol que tienen, y conocer la forma de planificar y programar de los mismos. En este sentido, casi la totalidad de los entrenadores de fútbol de formación de la región de Beja eran hombres. Estos resultados guardan relación con los datos publicados por el Instituto Portu- grado de acuerdo alto con los aspectos pertenecientes a la dimensión concepción de enseñanza, siendo uno de los más valorados el hecho de que el fútbol pueda ser un medio para educar a los jugadores. En esta línea, Merino, Sabirón \&Arraiz (2015) exponen que el entrenador debe asegurar que el aspecto educativo destaque en su identidad con respecto al competitivo. Además, independientemente del modelo deportivo que pueda ser adoptado, en edad escolar el deporte es una actividad que debe tener un fuerte componente educativo, y para que sea posible es necesario tener profesionales bien 
formados en todos los niveles deportivos (Feu, Ibáñez \& Gozalo, 2010). En este sentido, Olmedilla et al. (2004) consideran que quien tiene la clave del aspecto educativo en el deporte es el entrenador, siendo importante esta concepción del deporte como un medio educativo (Giménez,2003).

Tanto el factor referido a la importancia de que los jugadores se diviertan practicando fútbol como el relativo a que mediante la transmisión de valores se puede contribuir a la educación, se encontraron también entre los aspectos más valorados. Ambos son reforzados por Stafford (2010) al exponer que las intenciones de la práctica deportiva no se centran solo en el desarrollo de competencias motoras, sino que también tiene como objetivo construir cimientos basados en los valores y actitudes importantes para la alfabetización física y la adherencia a la práctica deportiva. También fue posible percibir un claro acuerdo mayoritario entre los entrenadores más jóvenes en relación con el hecho de que el fútbol podía ser un medio para transmitir valores educativos. Al tratarse de un tema actual, los entrenadores jóvenes tienen un mayor conocimiento sobre dicha problemática, pero debe ser una preocupación común a todos los entrenadores, pues, de acuerdo con Ortega et al. (2015), el entrenador es el elemento central para la transmisión de valores en el deporte.

Otro de los aspectos valorado positivamente, fue el referido a que todos los jugadores debían participar en las competiciones independientemente del resultado, ya que en ocasiones los entrenadores se dejan llevar por los aspectos resultadistas y pueden condicionar, aunque no de forma voluntaria, el hecho de experimentar la competición de una forma saludable. Por otra parte, los entrenadores atribuyeron una importancia relevante a los resultados obtenidos por el equipo en las competiciones. Al respecto, Merino et al. (2015), indican que la importancia atribuida al resultado y a la competitividad en el juego es claramente significativa en el contexto deportivo, al mismo tiempo que puede condicionar la participación y la instrucción del entrenador en cuanto al contenido.

Los resultados reportaron que los entrenadores mostraron un grado de acuerdo alto respecto al uso tanto de métodos directivos como de descubrimiento. Estos resultados, son similares a los encontrados por Abad et al. (2011), aunque no se asemejan a los encontrados por Lledó et al. (2014), quienes evidencian que los entrenadores emplean en su gran mayoría métodos directivos, donde el técnico es el que toma gran parte de las decisiones y el jugador sólo reproduce el esquema de acción definido por el entrenador.

Por otra parte, el aspecto en el que los entrenadores mostraron un grado bajo de acuerdo, fue el hecho referido a evaluar al final de la temporada. En este sentido, según Buceta (2004), el entrenador debe procurar evaluar la eficacia de su trabajo en las sesiones de entrenamiento regularmente, y de esta forma, sabrá cómo responden los deportistas a las exigencias, aquello que funciona de forma positiva y lo que debe cambiarse para optimizar la rentabilidad de los entrenamientos. Además, el seguimiento de los entrenamientos es de vital importancia para la reformulación de la planificación, proporcionando un feedback imprescindible para el entrenador (Labrincha \& Gaspar, 2004).

Por otro lado, en relación con los niveles de programación, se verificó que la mayoría de los encuestados afirmaron programar la temporada antes de que comenzara y también semanalmente. Estos resultados concuerdan con los obtenidos por Lledó \& Huertas (2012). Estos resultados se ven reforzados por la investigación realizada por Brink et al. (2014), ya que, para estos autores, la planificación semanal disminuye el margen de discordancia de la intensidad de entrenamiento favoreciendo el proceso de adaptación a la carga. Además, los entrenadores manifestaron un grado de acuerdo alto con la afirmación que hacía referencia a la intención de realizar una evaluación inicial de los jugadores estructurando la programación a partir de dicha evaluación. Teniendo en cuenta estos resultados, es importante, además, según Santos, Pereira \& Martinek (2018), que la programación se base en la experiencia y reflexión para aumentar de forma significativa la calidad de las experiencias vividas por los atletas.

La mayoría de los aspectos de la dimensión planificación y programación fueron valorados de forma positiva, sobre todo, el ítem referido al planteamiento de objetivos educativos. En este sentido, Del Bosque (2013) afirma que la excelencia deportiva no debe ser entendida como la conquista de títulos, sino como la búsqueda de una forma ética y estética de alcanzar el desarrollo personal y social. De esta manera, Feu et al. (2007), manifiestan que los entrenadores que trabajan en las categorías con jugadores más jóvenes presentan una mayor orientación hacia enfoques educativos y recreativos, dejando a un lado la orientación hacia el rendimiento.

El aprendizaje a través de la práctica deportiva representa el contexto con mayor relevancia en la promoción del desarrollo positivo de los jóvenes (Santos et al., 2018). De esta manera, y relacionándolo con las 
metodologías del entrenamiento, se vio reflejado que existía una clara valoración positiva por parte de la mayoría de los encuestados en relación con el hábito de trabajar los medios técnico-tácticos con oposición, dándoles más prioridad que a los físicos. Estos resultados no están en la línea de lo encontrado por Lledó et al. (2014), ya que los técnicos encuestados en este estudio tradujeron un cierto nivel de equilibrio entre los contenidos específicos, en particular la técnica y la táctica, y los contenidos generales relacionados con la condición física. $\mathrm{Al}$ respecto, tanto los entrenadores con más experiencia como los que tienen estudios universitarios relacionados con la Educación Física, afirman tener más claros los contenidos que tienen que trabajar en estas edades de formación.

Por otro lado, referente a los aspectos vinculados con las relaciones interpersonales, los encuestados confirmaron que era fácil para ellos motivar a sus jugadores. De acuerdo con Chelladurai, Kuga \& O’Bryant (1999), esto puede ser debido a que los entrenadores suelen tener cierta vocación por enseñar o entrenar. Estos resultados también están de acuerdo con la preocupación de Maestre et al. (2018), cuando afirman que los entrenadores que actúan en la formación de jóvenes deportistas deben conferir mayor relevancia a los aspectos que definen la dimensión personal. Por tanto, los resultados hallados pueden considerarse positivos, ya que la programación del entrenamiento para jóvenes deportistas tiene que preocuparse por desarrollar activamente relaciones de apoyo entre niño y adulto (FraserThomas, Côté \& Deakin, 2005). Además, según los encuestados, era fácil mantener relaciones cordiales con los padres de los jugadores, no tenían dificultades en controlar los entrenamientos y normalmente los jugadores eran asiduos a los mismos. Estos resultados se ven apoyados por Galatti et al. (2016) quienes exponen que el desarrollo del proceso de enseñanza del fútbol debe ser conducido por la figura de un entrenador, que necesita saber gestionar conocimientos intrapersonales, interpersonales y profesionales.

Por último, los entrenadores asumieron un grado de acuerdo notable con la intención de entrenar en categorías superiores en el futuro. Estos resultados, al igual que los obtenidos por Zapata, Antonio \& Palao (2015), parecen mostrar que la evolución del joven jugador en el proceso de formación no es una prioridad, sino que podría existir una preocupación por los resultados inmediatos. En este sentido, quizás, para poder huir de esta tendencia sería preferible tener los contenidos de trabajo definidos en función de una programación anual y mensual intentando cada semana cumplir lo que estaba previamente determinado, no dejando que el aspecto competitivo se adueñe del proceso.

\section{Conclusiones}

Teniendo en cuenta los objetivos planteados en el presente estudio se ha de destacar que el perfil del entrenador de fútbol base de Beja (Portugal) corresponde al siguiente: hombre de entre 31 y 40 años, con el duodécimo año de escolaridad completo (bachiller), trabajando por cuenta ajena, sin curso de entrenador y apenas con el Grado I o nivel básico.

Respecto a la concepción de la enseñanza del fútbol se ha de subrayar que los entrenadores lo consideran un medio para educar a los jugadores. Los encuestados entienden que es fundamental que los jugadores se diviertan practicando fútbol a la vez que se aboga por una transmisión de valores que contribuya a su educación. En este aspecto, los entrenadores más jóvenes son los que más creen en el fútbol como medio transmisor de valores. Por otro lado, los entrenadores están bastante de acuerdo con utilizar con frecuencia tanto los métodos directivos basados en el descubrimiento. Además, la consideración de que la competición sea solo un aspecto más en la formación deportiva está respaldada por todos los entrenadores, siendo los más jóvenes los más sensibles a que todos los jugadores deben participar independientemente del resultado.

En relación con los aspectos referidos a la forma de planificación y programación, gran parte de los entrenadores manifiestan que tienen costumbre de programar la temporada antes de que empiece y semanalmente. Por otra parte, la mayoría de los entrenadores dan más prioridad a los aspectos técnico-tácticos que a los físicos, presentando una mayor utilización de metodologías lúdicas, y existiendo una división entre los encuestados que plantean objetivos competitivos y los que persiguen fundamentalmente objetivos recreativos. No obstante, los entrenadores aseveran trabajar fundamentalmente objetivos educativos. Además, tanto los entrenadores con más experiencia como los que tienen estudios universitarios relacionados con la Educación Física, afirman tener más claros los contenidos que tienen que trabajar en estas edades de formación. Por último, la mayoría de los encuestados realizan una evaluación inicial de los jugadores al comienzo de la temporada y, a partir de ésta, elaboran la programación.

Las limitaciones encontradas a la hora de llevar a 
cabo este estudio hacen referencia, en primer lugar, al tipo de herramienta utilizada, ya que la información recogida a través del cuestionario puede variar en función de la honestidad e interés de los encuestados. Además, los cuestionarios que son cumplimentados vía telemática no permiten esclarecer cualquier tipo de duda que pudiera surgir durante dicho proceso.

En cuanto a las perspectivas de futuro, se ha de destacar el hecho de que sería conveniente seguir estudiando la figura del entrenador de fútbol base a través de estudios observacionales en los que se puedan confirmar, o no, los datos obtenidos en esta investigación. Otra línea de investigación, fundamentada en las evidencias de la literatura sobre la escasa formación de los técnicos del fútbol base, podría dirigirse al estudio de entrenadores cualificados con el fin de sacar a la luz los aspectos más destacados de éstos, y hacer propuestas de mejora de las competencias de los técnicos que se materialicen en la realización de formaciones de calidad basadas en los resultados obtenidos. Además, este estudio puede ser replicado en otras regiones y países con el objetivo de establecer el perfil del técnico en otros contextos y comparar las diferencias encontradas en las dimensiones estudiadas.

Para concluir, se ha de destacar que esta investigación podría ser de utilidad para la mejora de la formación de los entrenadores y de la enseñanza del fútbol, en la medida en que puede servir como estímulo para llevar a cabo las siguientes acciones al respecto: estimular a los entrenadores para que realicen el curso de entrenadores; fomentar una mayor utilización de métodos de enseñanza basados en la resolución de problemas y en el descubrimiento; concienciar sobre la importancia que tiene la enseñanza del fútbol en la formación y educación de los chicos y chicas; y mejorar algunos aspectos relacionados con la programación como los contenidos a trabajar en estas edades y la evaluación del proceso de enseñanza/entrenamiento.

\section{Referencias}

Abad, M.T. (2009). La formación del entrenador en el proceso de enseñanza- aprendizaje de jóvenes futbolistas. Tesis doctoral. Universidad de Huelva.

Abad, M.T., Benito, P. J., Giménez, F. J., \& Robles, J. (2011). La formación de los entrenadores de fútbol base en la provincia de Huelva. Cultura, Ciencia y Deporte, 6(18), 171-183.

Azpillaga, I., González, Ó., Irazusta, S., \& Arruza, J.A. (2012). Análisis y valoración de la influencia que ejerce el perfil formativo de los entrenadores en jóvenes futbolistas. Retos. Nuevas Tendencias en Educación Física, Deporte y Recreación, 22, 62-64.
Ballester, I., Fernández, R., \& Parra-Camacho, D. (2021).Adaptación y validación de una escala para la evaluación del desempeño profesional del entrenador de fútbol en base a su formación permanente, nivel de TIC y autoevaluación. Retos. Nuevas Tendencias en Educación Física, Deportey Recreación, 40, 272-280. doi: https: / /doi.org/10.47197/retos.v1i40.83157

Bettega, O., Scaglia, A., Nascimento, J., Ibáñez, S., \& Galatti, L. (2018). O ensino da tática e da técnica no futebol: concepção de treinadores das categorias de base. Retos. Nuevas Tendencias en Educación Física, Deporte y Recreación, 33, 112-117. doi: https: / /doi.org/10.47197/retos.v0i33.55503

Brink, M. S., Frencken, W. G. P., Jordet, G., \& Lemmink, K.A. P. M. (2014). Coaches' and Players' Perceptions of Training Dose: Not a Perfect Match. International Journal of Sports Physiology and Performance, 9, 497 -502. doi: 10.1123/IJSPP.2013-0009

Buceta, J. M. (2004). Estratégias Psicológicas para Treinadores de Desportistas Jovens. Dykinson. Madrid.

Cañadas, M., Ibáñez, S. J., García, J., Parejo, I., \& Feu, S. (2010). Importancia de la planificación en el entrenamiento deportivo: análisis del proceso de entrenamiento en minibasket, Wanceulen E.F.digital, 7, 52-64.

Cohen, J. (1988). Statistical power analysis for the behavioral sciences ( $2^{\mathrm{a}}$ ed.), Hillsdale, NewYork: Lawrence Erlbaum Associates, Publishers.

Chelladurai, P., Kuga, D. J., \& O’Bryant, C. P. (1999). Individual differences, perceived task characteristics, and preferences for teaching and coaching. Research Quarterly for Exercise and Sport, 70(2), 1-9. doi: http://dx.doi.org/10.1080/ 02701367.1999.10608035

Del Bosque, V. (2013). El Fútbol, Educación y Formación. Cultura, Cienciay Deporte, 22(8), 67-70.

Evans, M. B., McGuckin, M., Gainforth, H. L., Bruner, M.W., \& Côté, J. (2015). Coach development programmes to improve interpersonal coach behaviours: a systematic review using the re-aim framework. British Journal of Sports Medicine, 49(13), 871-877. doi:10.1136/bjsports-2015-094634

Feu, S., Ibáñez, S. J., \& Gozalo, M. (2007). Propiedades psicométricas de los cuestionarios EDD y EPD para evaluar el estilo de Planificación y decisión de los entrenadores. Revista de Psicología del Deporte, 16(2), 185-199.

Feu, S., Ibáñez, S. J., \& Gozalo, M. (2010). La formación inicial de los entrenadores de balonmano para la enseñanza del deporte en la edad escolar. Cultura, Ciencia y Deporte, 5(14), 109-117.

Fraser-Thomas, J. L., Côté, J., \& Deakin, J. (2005).Youth sport programs: an avenue to foster positive youth development. Physical Education and Sport Pedagogy, 10(1), 19-40. doi: http: / /dx.doi.org/10.1080/1740898042000334890

Galatti, L., Bettega, O., Montagner, P.C., \& Scaglia, A.J. (2016). Atribuições do professor e treinador de exporte: ensino do futebol em idade escolar. En S. Feu y M. Cañadas (Coords.). Innovaciones y aportaciones a la formación de entrenadores para el deporte en la edad escolar (pp. 239-252). Universidad de Extremadura. Editora Unicamp.

Giménez, F. J. (2003). La Formación del Entrenador en la Iniciación al Baloncesto. Sevilla:Wanceulen Editorial Deportiva S. L. 
Gutierrez, D. (2007). Modelo de intervencion para educar en valores a traves del futbol: una experiencia con entrenadores de futbol de la Comunidad de Madrid. Tesis doctoral inédita. Universidad Politécnica, Madrid.

Hernández, R., Fernández-Collado, C., \& Baptista, P. (2007).Metodología de la investigación (4a ed.). México: McGraw-Hill/ Interamericana Editores, S.A. de C.V.

Ibáñez, S. J. (2002). Los contenidos de enseñanza del Baloncesto en las categorías de formación. En S. J. Ibáñez \& M. Macías (Eds.), Novos horizontes para o treino do básquetbol (pp. 111-136). Lisboa: Ediçôes FMH.

Instituto Português do Desporto e Juventude, I.P. (2018). Infografia dosTécnicos de Desporto em Portugal. Departamento de Formação e Qualificação. Lisboa, Portugal.

Jiménez, C., López-Barajas, E., \& Pérez, R. (1997). Pedagogía experimental II. (Vol. I). Madrid: UNED.

Jones, R. (1992). Certified Football Association Coaches: Background,Training and Role Perceptions. British Journal of Physical Education, Research Suplement, 12, 2-6.

Kataoka, R., Vasenina, E., Loenneke, J., \& Buckner, S.L. (En prensa). Periodization:Variation in the Definition and Discrepancies in Study Design. Sports Medicine. Doi: 10.1007/s40279-02001414-5

Labrincha, J., \& Gaspar, P. (2004). Avaliação e controlo do treino em basquetebol: um estudo exploratório em treinadores. Revista Portuguesa de Ciências do Desporto, 4(2), 228.

Lledó, E., Martínez, G., \& Huertas, F. (2014). Perfil del entrenador de fútbol en la etapa escolar en escuelas de clubes de élite de la ComunitatValenciana. Cultura, Ciencia y Deporte, 9(25), 57-68.

Lledó, E., \& Huertas, F. (2012). Perfil del técnico de fútbol en escuelas de clubes de primera división en la ComunitatValenciana. Apunts.Educación Física y Deportes, 108, 35-45. doi: http:/ /dx.doi.org/10.5672/apunts.2014-0983.es.(2012/2).108.04

Macarro, J., Romero, C., \&Torres, J. (2010). Motivos de abandono de la práctica de actividad fisico-deportiva en los estudiantes de Bachillerato de la provincia de Granada. Revista de Educación, 353, 495-519.

Maestre, M., Garcés de los Fayos, E. J., Ortín, F. J., \& Hidalgo, M. D. (2018). El perfil del entrenador excelente en fútbol base. Un Estudio mediante Grupos Focales. Cuadernos de Psicología del Deporte, 18(3), 112-128.

Merino,A., Sabirón, F., \& Arraiz,A. (2015).Análisis del escenario de competición en fútbol prebenjamín: Un estudio de caso. Retos, Nuevas Tendencias en Educación Física, Deporte y Recreación, 28, 26-32. doi: https://doi.org/10.47197/retos.v0i28.34815

Nuviala, A., Leon, J.A., Galvez, J., \& Fernandez, A. (2007). Que actividades deportivas escolares queremos. Que tecnicos tenemos. Revista Internacional de Medicina y Ciencias de la Actividad Fisica y el Deporte, 25.

Nuviala,A., \& Nuviala, R. (2005). Abandono y continuidad de la práctica deportiva escolar organizada desde la perspectiva de los técnicos de una comarca aragonesa. Revista Internacional de Medicina y Ciencias de la Actividad Física y el Deporte, 20, 295 307.
Olmedilla, A., Ortín, F. J., Andréu, M. D., \& Lozano, F. J., (2004). Formación en Psicología para Entrenadores de Fútbol: Una Propuesta Metodológica. Revista de Psicología del Deporte, 13(2), 247-262.

Ortega, G., Abad, M.T. Durán, L. J., Franco, J., Giménez, F. J., Jiménez, P. J., \& Jiménez,A. C. (2015). Treinar Futebol:Ensinando Valores. Por uma Educação Real:Valores e Desporto. Madrid: Edições Gráficas Fundação Real Madrid.

Paixão, P.,Abad, M.T., \& Giménez, F. J. (2019). Diseño y validación de un cuestionario para estudiar la formación de entrenadores de fútbol base. Retos. Nuevas Tendencias en Educación Física, Deportey Recreación, 35, 294-300. doi: https://doi.org/10.47197/ retos.v0i35.63262

Santos, F., Côrte-Real, N., Regueiras, L., Dias, C., \& Fonseca,A. (2016). O papel do treinador do desenvolvimento positivo de jovens através do desporto: do que sabemos ao que precisamos saber. Revista Iberoamericana de Psicología del Ejercicio y el Deporte, 11(2), 289-296.

Santos, F., Pereira, P., \& Martinek,T.J. (2018). O papel do treinador no desenvolvimento positivo dos jovens através do desporto de competição: necessidades, desafios e recomendações. InV. Loureiro e N. Loureiro (Org.). Atividade Física e Desporto. Diversos Contextos de Intervenção (pp. 112-127). Instituto Politécnico de Beja.

Santos, S., Mesquita,I., Graça,A., \& Rosado,A. (2010). Coaches' perceptions of competence and acknowledgement of training needs related to professional competences. Journal of Sports Science and Medicine, 9, 62-70.

Siedentop, D. Hastie, H., \& Van Der Mars H. (2004). Complete Guide to Sport Education. Champaign, IL: Human Kinetics.

Sousa, C., Cruz, J., Torregrosa, M., Vilches, D., \& Viladrich, C. (2006). Evaluación conductual y programa de asesoramiento personalizado a entrenadores (PAPE) de deportistas jóvenes. Revista de Psicología del Deporte, 15(2), 263-278.

Stafford, I. (2010). Coaching for Long-Term Athlete Development:to improve participation and performance in sport.London. Sport England, Sports Coach.

Suchomel,T.J., Nimphius, S., Bellon, C.R., \& Stone, M.H. (2018). The importance of muscular strength: training considerations. Sports Medicine, 48(4), 765-785.

Tomczak, M., \& Tomcak, E. (2014). The need to report effect size estimates revisited. An overview of some recommended measures of effect size. Trends Sport Scicences, 1(21), 19-25.

Urbano-Arévalo, F.C., Mancha-Triguero, D., Gómez-Carmona, C.D., \& Gamonales, J.M. (2020). Influencia del perfil del entrenador en el diseño de tareas en fútbol-base. Estudio de casos. Retos. NuevasTendencias en Educación Física, Deporte y Recreación, 38, 204-212. doi: https://doi.org/10.47197/ retos.v38i38.74456

Viciana, J. (2002). Planificar en Educación Física. Barcelona: Inde.

Zapata, G.,Antonio, J., \& Palao, J. M. (2015). Planificación y Control del Entrenamiento de los Equipos de Fútbol en Categorías Juvenil y Tercera División Senior de la Región de Murcia (temporada 2012-2013), Kronos 14(1). 